\title{
ELECTROMAGNETIC AND GRAVITATIONAL RADIATION OF GRAVIATOMS
}

\author{
Yu.P. Laptev*and M.L. Fil'chenkov ${ }^{\dagger}$ \\ Institute of Gravitation and Cosmology, \\ Peoples' Friendship University of Russia \\ 6 Miklukho-Maklaya Street, Moscow 117198, Russia
}

October 6, 2018

\begin{abstract}
Graviatom existence conditions have been found. The graviatoms (quantum systems around mini-black-holes) satisfying these conditions contain the following charged particles: the electron, muon, tau lepton, wino, pion and kaon. Electric dipole and quadrupole and gravitational radiations are calculated for the graviatoms and compared with Hawking's mini-hole radiation.
\end{abstract}

Keywords: Graviatom; Electromagnetic and gravitational radiations

\section{Introduction}

A motion of micro-particles on scales larger than the Compton length is quantized in curved space-time. Kuchař has shown that the latter reduces to Schrödinger's equation with the Newtonian potential in a non-relativistic case [1]. The behaviour of charged particles in a centrally symmetric gravitational field was considered by DeWitt who obtained a so-called self-force, acting on the charge, apart from the Newtonian gravitational force [2].

A quantum-mechanical problem of electron motion in the gravitational field of a mini-hole was considered by Gaina who obtained hydrogen-like solutions [3].

The general case of charged particle motion in the Schwarzschild field was considered later taking account of DeWitt's self-force $[4,5,6]$. Primordial black holes (or mini-holes) can capture charged particles due to gravitational interaction. Bound quantum systems maintaining a charged particle in orbit around a mini-hole were called graviatoms [6].

In the present article, we shall derive graviatom existence conditions and calculate electromagnetic and gravitational radiations to be compared with Hawking's mini-hole radiation.

\section{Academic problem solution}

Schrödinger's equation for the graviatom [4]

$$
\frac{1}{r^{2}} \frac{d}{d r}\left[r^{2}\left(\frac{d R_{p l}}{d r}\right)\right]-\frac{l(l+1)}{r^{2}} R_{p l}+\frac{2 m}{\hbar^{2}}\left(E-\frac{m c^{2} r_{Q} r_{g}}{4 r^{2}}+\frac{m c^{2} r_{g}}{2 r}\right) R_{p l}=0
$$

describes a radial motion of a particle with the charge $Q$ and mass $m$ in the effective mini-hole potential, taking into account DeWitt's self-interaction, as follows

$$
U_{\mathrm{eff}}=-\frac{m c^{2} r_{r_{g}}}{2 r}+\frac{m c^{2} r_{Q} r_{g}}{4 r^{2}}+\frac{\hbar^{2} l(l+1)}{2 m r^{2}},
$$

*yplaptev@rambler.ru

†fmichael@mail.ru 
where $r_{g}=2 G M / c^{2}$ and $M$ are the mini-hole gravitational radius and mass, respectively, and $r_{Q}=Q^{2} /\left(m c^{2}\right)$ is the classical radius of the charged particle.

The solution to (1) has the form

$$
R_{\mathrm{pl}}=\text { const } \cdot \rho^{s} e^{-\rho / 2} F(-p, 2 s+2, \rho),
$$

where $F(-p, 2 s+2, \rho)$ is the confluent hypergeometric function,

$$
\begin{gathered}
\rho=\frac{2 \sqrt{-2 m E}}{\hbar} r, \quad s(s+1)=\frac{2 m A}{\hbar^{2}}+l(l+1), \\
A=\frac{m c^{2} r_{Q} r_{g}}{4}, \quad B=\frac{m c^{2} r_{g}}{2}, \quad p=n-s-1,
\end{gathered}
$$

$p=0,1,2, \ldots, \quad l \leq n, \quad n=1,2,3, \ldots \quad n$ and $l$ are the principal and orbital quantum numbers, respectively.

The energy spectrum of the charged particle captured by the mini-hole reads

$$
E=-\frac{2 B^{2} m}{\hbar^{2}} \frac{1}{\left[2 p+1+\sqrt{(2 l+1)^{2}+\frac{8 m A}{\hbar^{2}}}\right]^{2}} .
$$

If we take account of only the electromagnetic and gravitational interactions and neglect the strong one, determining nuclei sizes, the solutions (3) and (4) for the nuclei with the mass $m=2 Z m_{p}$ and charge $Q=Z e$ can be divided into two cases: the hydrogen-like one for light nuclei with $r_{g} r_{Q} / \lambda_{c}^{2} \ll 1$, whose energy spectrum is given by the formula

$$
E=-\frac{4 Z^{3} m_{p}^{3} G^{2} M^{2}}{\hbar^{2} n^{2}}
$$

where $\lambda_{c}=\hbar /(m c)$ is the Compton wavelength, $m_{p}$ is the proton mass and $G$ the gravitational constant, and that for Kratzer's potential, valid for heavy nuclei with $r_{g} r_{Q} / \hbar_{c}^{2} \gg 1$, whose energy spectrum takes the form

$$
E=-\frac{m c^{2} r_{g}}{4 r_{Q}}+\frac{\hbar c}{r_{Q}}\left(p+\frac{1}{2}\right) \sqrt{\frac{r_{g}}{2 r_{Q}}}+\frac{\hbar^{2}}{2 m r_{Q}^{2}}\left(l+\frac{1}{2}\right)^{2}-\frac{3 \hbar^{2}}{2 m r_{Q}^{2}}\left(p+\frac{1}{2}\right)^{2}-\frac{3 \hbar^{3}}{m^{2} c r_{Q}^{2} \sqrt{2 r_{g} r_{Q}}}\left(p+\frac{1}{2}\right)\left(l+\frac{1}{2}\right)^{2}
$$

where the second term describes oscillations, the third rotations, the fourth anharmonicity of oscillations and the fifth oscillation-rotation coupling. Below we shall call this case an oscillatory one.

\section{Graviatom existence conditions}

A graviatom can exist if the following conditions are fulfilled:

1) the geometrical condition $L>r_{g}+R$, where $L$ is the characteristic size of the graviatom, $R$ is that of a charged particle;

2) the stability condition: (a) $\tau_{\mathrm{gr}}<\tau_{H}$, where $\tau_{\mathrm{gr}}$ is the graviatom lifetime, $\tau_{H}$ is the mini-hole lifetime, (b) $\tau_{\mathrm{gr}}<\tau_{p}$, where $\tau_{p}$ is the particle lifetime (for unstable particles);

3) the indestructibility condition (due to tidal forces and Hawking's effect) $E_{d}<E_{b}$, where $E_{d}$ is the destructive energy, $E_{b}$ is the binding energy.

If we introduce the dimensionless quantity

$$
\alpha=\frac{G M m_{p}}{e^{2}}
$$

then the hydrogen-like case will correspond to

$$
\alpha \ll\left(\frac{\hbar c}{e^{2}}\right)^{2} \frac{m_{p}}{m Z^{2}}
$$

and the oscillatory one to

$$
\alpha \gg\left(\frac{\hbar c}{e^{2}}\right)^{2} \frac{m_{p}}{m Z^{2}} .
$$

The characteristic size of a hydrogen-like graviatom

$L=a_{B}^{g}$, where

$$
a_{B}^{g}=\frac{\hbar^{2}}{G M m^{2}}
$$


is Bohr's radius for the graviatom. The characteristic size of an oscillatory graviatom is $L=r_{Q}$. The characteristic size of a nucleus is $R=1.25 \cdot 10^{-13} A^{1 / 3} \mathrm{~cm}$, where $\mathrm{A}$ is the atomic weight of the nucleus.

The graviatom lifetime is

$$
\tau_{\mathrm{gr}}=\left(\frac{\hbar c}{e^{2}}\right)^{5}\left(\frac{m_{p}}{\alpha m}\right)^{4} \frac{\hbar}{Z^{2} m c^{2}}
$$

the mini-hole lifetime is

$$
\tau_{H}=\frac{15360 \pi G^{2} M^{3}}{\hbar c^{4}}
$$

The lifetime of an unstable particle is

$$
\tau_{p}=\frac{\hbar}{\Gamma}
$$

where $\Gamma$ is the natural linewidth.

The destructive energy is $E_{d}=\left\{U_{t}, E_{H}\right\}$, with the tidal energy

$$
U_{t}=\frac{G M m R}{r^{2}}
$$

and Hawking's radiation energy

$$
E_{H}=\frac{b}{8 \pi} \frac{\hbar c}{e^{2}} \frac{m_{p} c^{2}}{\alpha}
$$

where $\hbar \omega_{m}=b k T$, according to Wien's displacement law, and $b=2.822$. is

The binding energy is $E_{b}=\left\{E_{W}, I_{\text {ion }}\right\}$, where $E_{W}$ is the nuclear binding energy. The graviatom ionization energy

$$
I_{\text {ion }}=\frac{m^{3} e^{4} \alpha^{2}}{2 n^{2} \hbar^{2} m_{p}^{2}} .
$$

In terms of $\alpha$, the graviatom existence conditions read

$$
\begin{gathered}
\alpha<\frac{1}{\sqrt{2}} \frac{\hbar c}{e^{2}} \frac{m_{p}}{m} \quad\left(a_{B}^{g}>r_{g}, \quad R \ll r_{g}\right), \\
\alpha>\left(\frac{\hbar c}{e^{2}}\right)^{8 / 7} \sqrt[7]{\frac{m_{p}^{7}}{15360 \pi Z^{2} m_{p l}^{2} m^{5}}} \quad\left(\tau_{g r}<\tau_{H}\right),
\end{gathered}
$$

where $m_{\mathrm{pl}}=\sqrt{\hbar c / G}$ is the Planck mass,

$$
\begin{gathered}
\alpha>\left(\frac{\hbar c}{e^{2}}\right)^{5 / 4} \frac{m_{p}}{m} \sqrt[4]{\frac{\Gamma}{m c^{2}}} \frac{1}{\sqrt{Z}} \quad\left(\tau_{g r}<\tau_{p}\right), \\
\alpha>\frac{b}{8 \pi} \frac{\hbar c}{e^{2}} \frac{m_{p} c^{2}}{E_{W}} \quad\left(E_{H}<E_{W}\right), \\
\alpha>\frac{\hbar c}{e^{2}} \frac{m_{p}}{m} \sqrt[3]{\frac{b n^{2}}{4 \pi}} \quad\left(E_{H}<I_{\mathrm{ion}}\right), \\
\alpha>\left(\frac{\hbar c}{e^{2}}\right)^{4 / 3} \frac{m_{p}}{m} \sqrt[3]{\frac{E_{W} e^{2}}{m^{2} c^{4} R}} \quad\left(U_{t}<E_{W}\right), \\
\alpha<\left(\frac{\hbar c}{e^{2}}\right)^{2} \frac{m_{p}}{m} \frac{e^{2}}{2 n^{2} m c^{2} R} \quad\left(U_{t}<I_{\mathrm{ion}}\right) .
\end{gathered}
$$

The conditions (17)-(23) prove to be fulfilled only for hydrogen-like graviatoms, i.e., if the condition (8) is satisfied. Moreover, it is the graviatoms containing leptons and mesons with $Z=1$ that satisfy these conditions. On the contrary, neither the graviatoms containing hadrons nor atomic nuclei can satisfy them for both a hydrogen-like case (hadrons, light nuclei) and an oscillatory one (heavy nuclei). The charged particles able to be constituents of the graviatom are: the electron, muon, tau lepton, wino, pion and kaon.

The graviatom existence conditions restrict the quantity $\alpha$ (proportional to the mini-hole mass $M$ ) within a narrow corridor of its values depending on the charged particle mass $m$ (see Fig.1). 


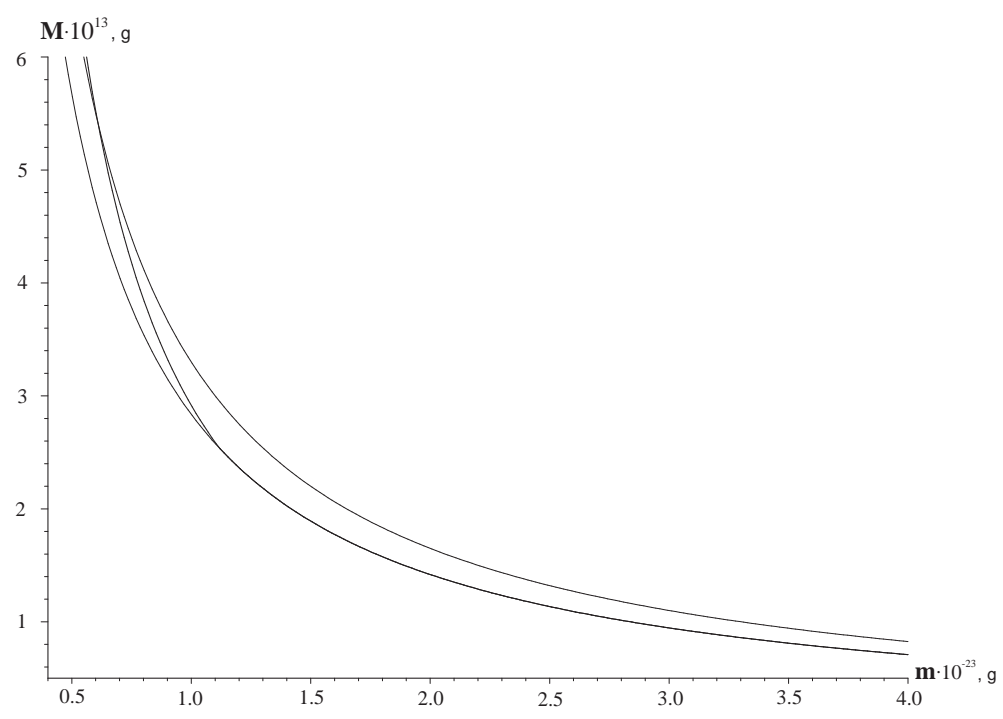

Figure 1: The dependence of mini-hole masses on the charged particle masses satisfying the graviatom existence conditions. The light curves indicate the range of values related to the geometrical condition (the upper curve) and to Hawking's effect ionization one (the lower curve). The heavy curve is related to the particle stability condition $\left(\tau_{p}=10^{-22} \mathrm{~s}\right)$.

\section{Graviatom radiation}

The intensity of the electric dipole radiation of a particle with mass $m$ and charge $e$ in the gravitational field of a mini-hole reads

$$
I_{f i}^{d}=\frac{2 \hbar e^{2} \omega_{i f}^{3} f_{i f}}{m c^{3}}
$$

where $\omega_{i f}=\left(E_{i}-E_{f}\right) / \hbar$ is the frequency of the transition $i \rightarrow f$ and $f_{i f}$ is the oscillator strength [7]. The condition of radiation being dipole is

$$
\alpha<\frac{m_{p}}{m} \frac{\hbar c}{e^{2}}
$$

which almost coincides with the geometrical condition (17) to be satisfied for the graviatoms under consideration.

The electric dipole radiation intensity for the hydrogen-like graviatom performing the transition $2 p \rightarrow 1 s$ is

$$
I_{12}^{d}=\frac{2 \hbar e^{2} \omega_{21}^{3}}{m c^{3}} f_{2 p \rightarrow 1 s},
$$

with the oscillator strength

$$
f_{2 p \rightarrow 1 s}=\frac{2^{13}}{3^{9}}=0.4162
$$

and the transition energy

$$
\hbar \omega_{12}=\frac{3 \alpha^{2} e^{4} m^{3}}{8 m_{p}^{2} \hbar^{2}} .
$$

Finally we obtain

$$
I_{12}^{d}=\frac{2^{5} \alpha^{6} e^{12} m^{8}}{3^{6} c^{3} \hbar^{8} m_{p}^{6}} .
$$

The electric dipole radiation intensity for the transition $3 p \rightarrow 1 s$ is

$$
I_{13}^{d}=\frac{2 \hbar e^{2} \omega_{31}^{3}}{m c^{3}} f_{3 p \rightarrow 1 s}
$$

with the oscillator strength

$$
f_{3 p \rightarrow 1 s}=\frac{3^{4}}{2^{10}}=0.0791
$$


Table 1. Graviatom parameters for the electron, muon and tau lepton.

\begin{tabular}{|c|c|c|c|c|c|c|}
\hline & \multicolumn{6}{|c|}{ Charged particles } \\
\hline & \multicolumn{2}{|c|}{ e } & \multicolumn{2}{|c|}{$\mu$} & \multicolumn{2}{|c|}{$\tau$} \\
\hline \multirow{3}{*}{$\begin{array}{l}m c^{2}, \mathrm{MeV} \\
\tau_{p}, \mathrm{~s}\end{array}$} & \multirow{2}{*}{\multicolumn{2}{|c|}{$\begin{array}{l}0.511 \\
\infty\end{array}$}} & \multirow{2}{*}{\multicolumn{2}{|c|}{$\begin{array}{l}105.659 \\
2.200 \mathrm{E}-6\end{array}$}} & \multirow{2}{*}{\multicolumn{2}{|c|}{$\begin{array}{l}1.777 \mathrm{E}+3 \\
2.900 \mathrm{E}-13\end{array}$}} \\
\hline & & & & & & \\
\hline & $\min$ & $\max$ & $\min$ & $\max$ & $\min$ & $\max$ \\
\hline $\mathrm{M}, \mathrm{g}$ & $3.12 \mathrm{E}+17$ & $3.62 \mathrm{E}+17$ & $1.51 \mathrm{E}+15$ & $1.75 \mathrm{E}+15$ & $9.96 \mathrm{E}+13$ & $1.04 \mathrm{E}+14$ \\
\hline$r_{g}, \mathrm{~cm}$ & $4.69 \mathrm{E}-11$ & $5.46 \mathrm{E}-11$ & $2.27 \mathrm{E}-13$ & $2.64 \mathrm{E}-13$ & $1.35 \mathrm{E}-14$ & $1.57 \mathrm{E}-14$ \\
\hline$a_{B}^{g}, \mathrm{~cm}$ & $5.46 \mathrm{E}-11$ & $6.35 \mathrm{E}-11$ & $2.64 \mathrm{E}-13$ & $3.07 \mathrm{E}-13$ & $1.57 \mathrm{E}-14$ & $1.83 \mathrm{E}-14$ \\
\hline$\hbar \omega_{12}, \mathrm{MeV}$ & 0.071 & 0.096 & 14.64 & 19.81 & 246.2 & 333.2 \\
\hline$\hbar \omega_{13}, \mathrm{MeV}$ & 0.084 & 0.114 & 17.35 & 23.48 & 291.8 & 394.9 \\
\hline$I^{d}(2 p \rightarrow 1 s), \operatorname{erg} s^{-1}$ & $1.02 \mathrm{E}+10$ & $2.55 \mathrm{E}+10$ & $4.39 \mathrm{E}+14$ & $1.09 \mathrm{E}+15$ & $1.24 \mathrm{E}+17$ & $3.08 \mathrm{E}+17$ \\
\hline$I^{d}(3 p \rightarrow 1 s), \operatorname{erg} s^{-1}$ & $3.25 \mathrm{E}+9$ & $8.05 \mathrm{E}+9$ & $1.39 \mathrm{E}+14$ & $3.44 \mathrm{E}+14$ & $3.93 \mathrm{E}+16$ & $9.74 \mathrm{E}+16$ \\
\hline$I^{q}(3 d \rightarrow 1 s), \operatorname{erg} s^{-1}$ & $1.33 \mathrm{E}+8$ & $4.47 \mathrm{E}+8$ & $5.70 \mathrm{E}+12$ & $1.91 \mathrm{E}+13$ & $1.61 \mathrm{E}+15$ & $5.41 \mathrm{E}+15$ \\
\hline$I^{g}(3 d \rightarrow 1 s), \operatorname{erg} s^{-1}$ & $1.11 \mathrm{E}+10$ & $4.36 \mathrm{E}+10$ & $4.75 \mathrm{E}+14$ & $1.85 \mathrm{E}+15$ & $1.34 \mathrm{E}+17$ & $5.24 \mathrm{E}+17$ \\
\hline$E_{H}, \mathrm{MeV}$ & 0.081 & 0.094 & 16.78 & 19.52 & 282.2 & 328.3 \\
\hline$P_{H}, \operatorname{erg} s^{-1}$ & $2.63 \mathrm{E}+10$ & $3.56 \mathrm{E}+10$ & $1.13 \mathrm{E}+15$ & $1.52 \mathrm{E}+15$ & $3.18 \mathrm{E}+17$ & $4.31 \mathrm{E}+17$ \\
\hline
\end{tabular}

Table 2. Graviatom parameters for the wino, pion and kaon.

\begin{tabular}{|c|c|c|c|c|c|c|}
\hline & \multicolumn{6}{|c|}{ Charged particles } \\
\hline & \multicolumn{2}{|c|}{$\tilde{W}$} & \multicolumn{2}{|c|}{$\pi$} & \multicolumn{2}{|c|}{$\mathrm{K}$} \\
\hline \multirow{3}{*}{$\begin{array}{l}m c^{2}, \mathrm{MeV} \\
\tau_{p}, \mathrm{~s}\end{array}$} & \multirow{2}{*}{\multicolumn{2}{|c|}{$\begin{array}{l}8.000 \mathrm{E}+5 \\
5.000 \mathrm{E}-10\end{array}$}} & \multirow{2}{*}{\multicolumn{2}{|c|}{$\begin{array}{l}139.568 \\
2.600 \mathrm{E}-8\end{array}$}} & \multirow{2}{*}{\multicolumn{2}{|c|}{$\begin{array}{l}493.994 \\
1.200 \mathrm{E}-8\end{array}$}} \\
\hline & & & & & & \\
\hline & $\min$ & $\max$ & $\min$ & $\max$ & $\min$ & $\max$ \\
\hline $\mathrm{M}, \mathrm{g}$ & $1.99 \mathrm{E}+11$ & $2.31 \mathrm{E}+11$ & $1.14 \mathrm{E}+15$ & $1.33 \mathrm{E}+15$ & $3.22 \mathrm{E}+14$ & $3.75 \mathrm{E}+14$ \\
\hline$r_{g}, \mathrm{~cm}$ & $2.99 \mathrm{E}-17$ & $3.49 \mathrm{E}-17$ & $1.72 \mathrm{E}-13$ & $1.99 \mathrm{E}-13$ & $4.86 \mathrm{E}-14$ & $5.65 \mathrm{E}-14$ \\
\hline$a_{B}^{g}, \mathrm{~cm}$ & $3.49 \mathrm{E}-17$ & $4.06 \mathrm{E}-17$ & $1.99 \mathrm{E}-13$ & $2.33 \mathrm{E}-13$ & $5.65 \mathrm{E}-14$ & $6.57 \mathrm{E}-14$ \\
\hline$\hbar \omega_{12}, \mathrm{MeV}$ & $1.11 \mathrm{E}+5$ & $1.50 \mathrm{E}+5$ & 19.34 & 26.17 & 68.44 & 92.62 \\
\hline$\hbar \omega_{13}, \mathrm{MeV}$ & $1.31 \mathrm{E}+5$ & $1.78 \mathrm{E}+5$ & 22.92 & 31.02 & 81.12 & 109.78 \\
\hline$I^{d}(2 p \rightarrow 1 s), \operatorname{erg} s^{-1}$ & $2.52 \mathrm{E}+22$ & $6.24 \mathrm{E}+22$ & $7.66 \mathrm{E}+14$ & $1.90 \mathrm{E}+15$ & $9.60 \mathrm{E}+15$ & $2.38 \mathrm{E}+16$ \\
\hline$I^{d}(3 p \rightarrow 1 s)$, erg $s^{-1}$ & $7.96 \mathrm{E}+21$ & $1.97 \mathrm{E}+22$ & $2.42 \mathrm{E}+14$ & $6.01 \mathrm{E}+14$ & $3.04 \mathrm{E}+15$ & $7.53 \mathrm{E}+15$ \\
\hline$I^{q}(3 d \rightarrow 1 s), \operatorname{erg} s^{-1}$ & $3.27 \mathrm{E}+20$ & $1.10 \mathrm{E}+21$ & $9.95 \mathrm{E}+12$ & $3.34 \mathrm{E}+13$ & $1.25 \mathrm{E}+14$ & $4.18 \mathrm{E}+14$ \\
\hline$I^{g}(3 d \rightarrow 1 s)$, erg $s^{-1}$ & $2.72 \mathrm{E}+22$ & $1.06 \mathrm{E}+23$ & $8.29 \mathrm{E}+14$ & $3.23 \mathrm{E}+15$ & $1.04 \mathrm{E}+16$ & $4.05 \mathrm{E}+16$ \\
\hline$E_{H}, \mathrm{MeV}$ & $1.27 \mathrm{E}+5$ & $1.48 \mathrm{E}+5$ & 22.16 & 25.78 & 78.44 & 91.25 \\
\hline$P_{H}, \operatorname{erg~s}^{-1}$ & $6.45 \mathrm{E}+22$ & $8.73 \mathrm{E}+22$ & $1.96 \mathrm{E}+15$ & $2.66 \mathrm{E}+15$ & $2.46 \mathrm{E}+16$ & $3.33 \mathrm{E}+16$ \\
\hline
\end{tabular}


Table 3. Relations valid for all graviatoms

\begin{tabular}{|c|l|l|}
\hline \multirow{2}{*}{ Relations } & \multicolumn{2}{|c|}{ Values } \\
\cline { 2 - 3 } & $\min$ & $\max$ \\
\hline$I^{g}(3 d \rightarrow 1 s) / I^{q}(3 d \rightarrow 1 s)$ & 83.295 & 96.899 \\
$I^{g}(3 d \rightarrow 1 s) / I^{d}(2 p \rightarrow 1 s)$ & 1.082 & 1.703 \\
$I^{g}(3 d \rightarrow 1 s) / I^{d}(3 p \rightarrow 1 s)$ & 3.419 & 5.383 \\
$I^{d}(2 p \rightarrow 1 s) / P_{H}$ & 0.390 & 0.715 \\
$\hbar \omega_{12} / E_{H}$ & 0.872 & 1.015 \\
$\sqrt{M m} / m_{p l}$ & 0.780 & 0.841 \\
\hline
\end{tabular}

and the transition energy

$$
\hbar \omega_{31}=\frac{2^{2} \alpha^{2} e^{4} m^{3}}{3^{2} \hbar^{2} m_{p}^{2}}
$$

Finally we obtain

$$
I_{13}^{d}=\frac{\alpha^{6} e^{14} m^{8}}{2^{3} 3^{2} c^{3} \hbar^{8} m_{p}^{6}} .
$$

Hence it follows

$$
\frac{I_{12}^{d}}{I_{13}^{d}}=\frac{2^{8}}{3^{4}}=3.161, \quad \frac{\omega_{21}}{\omega_{31}}=\frac{3^{3}}{2^{5}}=0.844 .
$$

The electric quadrupole radiation intensity for the transition $3 d \rightarrow 1 s$ is

$$
I_{13}^{q}=\frac{6 \hbar e^{2} \omega_{31}^{3}}{m c^{3}} f_{3 d \rightarrow 1 s}
$$

with the oscillator strength

$$
f_{3 d \rightarrow 1 s}=\frac{3^{7}}{2^{16}}\left(\frac{M m}{m_{p l}^{2}}\right)^{2},
$$

where the transition energy $\omega_{31}$ is given by formula (32). Finally we obtain

$$
I_{13}^{q}=\frac{\alpha^{8} e^{18} m^{10}}{2^{3} 3^{4} c^{5} \hbar^{10} m_{p}^{8}} .
$$

The gravitational radiation intensity for the graviatom performing the transition $3 d \rightarrow 1 s$ reads

$$
I_{13}^{g}=\frac{6 \hbar G M \omega_{31}^{3}}{c^{3}} f_{3 d \rightarrow 1 s},
$$

where the oscillator strength and the transition energy are given by formulae (32) and (35) respectively. Finally we obtain

$$
I_{13}^{g}=\frac{\alpha^{9} e^{18} m^{11}}{2^{3} 3^{4} c^{5} \hbar^{10} m_{p}^{9}} .
$$

The mini-hole creates particles near its horizon which can ionize graviatoms and split nuclei being their constituents. Hawking's effect power is given by the formula

$$
P_{H}=\frac{1}{15360 \pi \alpha^{2}}\left(\frac{\hbar c}{e^{2}}\right)^{2} \frac{\left(m_{p} c^{2}\right)^{2}}{\hbar} .
$$

Hawking's energy is

$$
E_{H}=\frac{b}{8 \pi \alpha} \frac{\hbar c}{e^{2}} m_{p} c^{2}
$$


The mini-hole mass $M$ is diminishing due to Hawking's effect evaporation, i.e.

$$
M_{f}=\sqrt[3]{M_{i}^{3}-\frac{\hbar c^{4}}{5120 \pi H G^{2}}}
$$

where $M_{f}$ and $M_{i}$ are the final and initial mini-hole masses respectively, $H$ is the Hubble parameter. $M_{f}=0$ corresponds to the minihole mass $M_{f}$ to have evaporated for the Universe's lifetime $T$

$$
M_{i}=\sqrt[3]{\frac{\hbar c^{4}}{5120 \pi H G^{2}}}
$$

which gives $M_{i}=4.38 \cdot 10^{14} g$ for $H=65 \mathrm{kms}^{-1} \mathrm{Mpc}^{-1} \quad\left(H=\frac{1}{T}\right)$.

Tables 1 and 2 present the graviatom parameters: the mini-hole and charge particle masses satisfying the graviatom existence conditions, the energies and intensities of the electromagnetic, gravitational and Hawking's radiations. Besides, unstable particle lifetimes (for the wino see [8]) and Bohr's graviatom radii are indicated.

The mini-holes being constituents of the graviatoms are formed due to Jeans' gravitational instability at the times about $\frac{r_{g}}{c}=10^{-27} \div 10^{-21} s$ from the initial singularity. The mini-hole masses for the graviatoms involving electrons, muons and pions exceed the value of $4.38 \cdot 10^{14} \mathrm{~g}$, which means that it is possible for such graviatoms to have existed up to now [9].

Table 3 presents relations valid for all graviatoms: the gravitational-to-electromagnetic radiation intensity ratios, the dipole-to-Hawking radiation ratio as well as the quantity equal to the square root of $\frac{G M m}{\hbar c}=0.608 \div 0.707$. The latter is a gravitational equivalent of the fine structure constant. The gravitational radiation intensities two orders exceed the electromagnetic ones. The graviatom dipole radiation energies and intensities have proved to be comparable with those for Hawking's effect of the mini-holes being constituents of the graviatoms. The gravitational equivalent of the fine structure constant does not exceed unity, thus the perturbation theory remains valid.

\section{Conclusion}

We have considered the graviatom existence conditions proving to be satisfied for charged leptons and mesons but not baryons (protons and nuclei). The baryon sizes appear to exceed the mini-hole gravitational radii which means that neither a hydrogen-like nor oscillatory case can take place, i.e., stable graviatoms with baryon constituents become impossible. Instead of them, there occurs a so-called quantum accretion of baryons onto a mini-hole. The internal structure of the baryons, consisting of quarks and gluons, should be taken into account. The whole problem is solvable within the framework of quantum chromodynamics and quantum electrohydrodynamics. The radiation of

baryons and quarks is also worth consideration later on. In the future, also of interest is to consider the graviatoms as sources of the electromagnetic background radiation and their possible contribution to dark matter.

\section{References}

[1] K. Kuchař, Phys. Rev. D 22, 1285 (1980).

[2] C.M DeWitt and B.S. DeWitt, Physics 1, 3 (1964).

[3] A.B. Gaina, PhD Thesis, Moscow State University, Moscow, 1980.

[4] M.L. Fil'chenkov, Astron. Nachr. 311, 223 (1990).

[5] M.L. Fil'chenkov, Izvestiya Vuzov, Fizika No. 7, 75 (1998).

[6] Yu.P. Laptev and M.L. Fil'chenkov, 41st All-Russian Conf. on Problems of Mathematics, Informatics, Physics and Chemistry. Physical Sections. Abstracts, Moscow: Peoples' Friendship University of Russia, 2005, p. 60.

[7] H.A. Bethe and E.E. Salpeter, "Quantum Mechanics of One- and Two-Electron Atoms", Springer-Verlag, Berlin, 1957.

[8] M. Sher, hep-th/9504257.

[9] V.P. Frolov, in "Einstein Col." 1975-1976, Nauka, Moscow, 1978, p. 82-151. 